DOI: https://doi.org/10.46296/yc.v4i7edespdic.0071

\title{
DIAGNÓSTICO DE LA GESTIÓN DE VENTAS DE VINOS DEL HOTEL SOL CAYO COCO
}

\section{DIAGNOSIS OF THE WINE SALES MANAGEMENT OF THE SOL CAYO COCO HOTEL}

\author{
Ramos-Martínez Arley*; García-Vergara Zenia 2; Ramos-López Lisandro³; Guerrero- \\ Hidalgo Raisa ${ }^{4}$ \\ ${ }^{1}$ Hotel Sol Cayo Coco. Ciego de Ávila, Cuba. Orcid: https://orcid.org/0000-0003-1171- \\ 2583 \\ 2 Profesor Instructor del departamento Tecnología y Ciencias de los Alimentos, \\ Universidad de Camagüey Ignacio Agramonte Loynaz. Camagüey, Cuba. Orcid: \\ https://orcid.org/0000-0001-6730-6758 \\ 3 Profesor Asistente del departamento Tecnología y Ciencias de los Alimentos, \\ Universidad de Camagüey Ignacio Agramonte Loynaz. Camagüey, Cuba. Orcid: \\ https://orcid.org/0000-0003-2564-7671 \\ ${ }^{4}$ Profesor Auxiliar del departamento Tecnología y Ciencias de los Alimentos, \\ Universidad de Camagüey Ignacio Agramonte Loynaz. Camagüey, Cuba. Orcid: \\ https://orcid.org/0000-0002-8232-3916
}

*Correo: zenia.garcia@reduc.edu.cu

\section{Resumen}

El servicio del hotel Sol Cayo Coco funciona bajo el régimen de Todo Incluido, en el departamento de Alimentos y Bebidas existe una gama de productos de alta calidad y Premium que se oferta a los clientes en efectivo para satisfacer gustos más exigentes y que conforman el llamado Servicio Extracarta. Dentro este se cuenta principalmente la oferta de vinos del Restaurante Don Diego y la oferta de espirituosos y cervezas en el Lobby Bar. Se concede gran importancia a la venta de estos productos por constituir una fuente de ingresos directa dentro de la oferta gastronómica y contribuir a la eficiencia económica del hotel y a la satisfacción de los clientes. El objetivo de la presente investigación es diagnosticar la gestión de ventas de vinos dentro de los productos extracartas del restaurante especializado Don Diego. Se realizó una investigación aplicada, de campo, no experimental y transversal; para esto se emplearon métodos del nivel empírico como la observación en los puntos de venta y el análisis de documentos entre los que se encuentran los estándares de presentación y los documentos del departamento económico y el almacén; y del nivel teórico como el análisis síntesis, la inducción deducción y el histórico lógico. Se determinaron una serie de medidas para el rediseño de una carta con propuestas más atractivas con el fin de satisfacer a los clientes.

Palabras clave: vino; extracarta; servicios; ventas.

\begin{abstract}
The service of the Sol Cayo Coco hotel works under the All-Inclusive regime, in the Food and Beverages department there is a range of high quality and Premium products that are offered to customers in cash to satisfy the most demanding tastes and that make up the so-called Extra letter service. This includes mainly the offer of wines from the Don Diego Restaurant and the offer of spirits and beers in the Lobby Bar. Great importance is attached to the sale of these products as they constitute a direct source of income within the gastronomic offer and contribute to the economic efficiency of the hotel and customer satisfaction. The objective of this research is to diagnose the management of wine sales within the extra-menu products of the specialized
\end{abstract}

Información del manuscrito:

Fecha de recepción: 05 de noviembre de 2020.

Fecha de aceptación: 14 de diciembre de 2020.

Fecha de publicación: 21 de diciembre de 2020. 
restaurant Don Diego. An applied, field, non-experimental and transversal research was carried out; For this, empirical level methods such as observation at the points of sale and the analysis of documents were used, among which are the presentation standards and the documents of the economic department and the warehouse; and from the theoretical level such as synthesis analysis, deduction induction and logical history. A series of measures were determined for the redesign of a letter with more attractive proposals to satisfy customers.

Keywords: wine; extra letter; services; sale.

\section{Introducción}

Resulta ya innegable en el tránsito de siglos advertir el crecimiento cualitativo y cuantitativo del turismo en el ámbito mundial. El lanzamiento de nuevos destinos y la reconversión de otros ya existentes, la aparición de un cuerpo de reglamentos, convenciones y normas jurídicas, las exigentes campañas publicitarias y la consolidación de acercamientos científicos al estudio de este fenómeno social (Venegas, 2005).

Nieto, Román, Bonillo, y Paulova (2016) plantean que, el turismo es una de las actividades económicas que ha sufrido una mayor expansión en las últimas décadas. Si nos remontamos a mediados del siglo $\mathrm{XX}$, podemos constatar que el turismo internacional era una actividad meramente simbólica, con solo 25 millones de turistas a nivel global en el año 1950. De hecho, la evolución ha sido vertiginosa, pues en el año 2000 se alcanzaron los 678 millones de turistas, ascendiendo a 950 millones en 2010. Además, según las estimaciones de la Organización Mundial del Turismo se espera que para 2030 se superen los 1.800 millones de turistas.

Cuba es uno de los destinos de mayor crecimiento en la actualidad en el Caribe, es a su vez un destino turístico interesante y complejo de analizar, en particular desde el punto de vista geográfico, por: su cantidad y variedad de atractivos y recursos de interés turístico; su ubicación privilegiada en el contexto geográfico del Caribe Insular y Centroamérica, su importancia geopolítica durante las últimas décadas, a partir del triunfo de la Revolución el primero de Enero del año 1959; y sus históricas y contradictorias relaciones con los EE.UU (Ruiz, 2014).

El Servicio Gastronómico es una de las actividades clave de las 
instalaciones hoteleras cubanas al satisfacer necesidades básicas. Las áreas de servicios son puntos de contacto directo, donde se dan los momentos de la verdad en la prestación del servicio, aspecto que las hace muy vulnerables en la percepción de la calidad por parte del cliente.

El Hotel Sol Cayo Coco cuenta con un producto 4 estrellas en contrato de administración con la firma Sol Meliá, una instalación enfocada al turismo de familia interesada en disfrutar del ambiente. Aunque todo el servicio del hotel funciona bajo el régimen de Todo Incluido, en el departamento de $A+B$ existe una gama de productos de alta calidad y productos Premium que se oferta a los clientes en efectivo para satisfacer gustos más exigentes y que conforman el llamado Servicio Extracarta. Dentro del servicio de Extracartas se cuenta principalmente la oferta de vinos del Restaurante Especializado Don Diego. El hotel concede gran importancia a la venta de estos productos por constituir una fuente de ingresos directa dentro de la oferta gastronómica y contribuir tanto a la eficiencia económica del hotel como a la satisfacción de los clientes.

Por parte de la dirección del hotel se ha detectado un decrecimiento en las ventas de las Bebidas Extracartas que ha llevado al incumplimiento de los presupuestos elaborados para los ingresos por este concepto. El objetivo de la presente investigación es diagnosticar la gestión de ventas de vinos dentro de los productos extracartas del restaurante especializado Don Diego.

\section{Materiales y métodos}

Se realiza una investigación aplicada, de campo, no experimental y transversal en la que emplearon como métodos de investigación, entre los del nivel teórico: el histórico-lógico, el análisis-síntesis, la abstracciónconcreción: para la determinación y ordenamiento de los antecedentes del tema, fundamentando el objeto y campo de la investigación así como en la interpretación de la información aportada por los métodos empíricos, para realizar el análisis de la oferta $y$ el enfoque de sistema en el tratamiento de las 
interrelaciones de todos los elementos que inciden en la gestión de la oferta extracarta y en la satisfacción del cliente. Dentro de los métodos del nivel empírico se trabajó con la observación en los puntos de venta involucrados y el análisis de documentos entre los que se encuentran los estándares de presentación y los documentos del Departamento Económico y Almacén.

Se realizó un diagnóstico de la carta de vino teniendo en cuenta las características gestión de compras y reposición; las condiciones de almacenamiento y conservación; el diseño y contenido de la carta de vinos; y el servicio.

\section{Resultados y discusión}

Diagnóstico de gestión de compra y venta de vinos

En el hotel se detectaron problemas en el almacén donde hay vinos en existencia que ya no están aptos para la venta pues su ciclo de vida ya terminó lo que representa pérdida de recursos y gasto de mantenimiento de un producto ya deteriorado, esto está dado por una compra no acertada y una mala rotación del producto, así como se evidencia falta de conocimientos por parte del personal que atiende la actividad en los almacenes.

Según Gil, Berenguer y Ruiz (2009) el contenido de la carta de vinos y la presencia de personal formado en vinos se constituyen en los dos instrumentos básicos de promoción de los vinos en el restaurante. En general, los restaurantes de categoría superior cuentan con recursos técnicos (conocimientos, "saber hacer", especialización profesional), institucionales (menciones, inclusión en guías gastronómicas de prestigio) y físicos (infraestructura del restaurante, ubicación y decoración) que permiten su diferenciación. No obstante, dos van a ser los recursos que con más asiduidad utilizan los restaurantes como herramienta de diferenciación: la gestión de la carta de vinos y la presencia de un sumiller o un profesional especializado en vinos. Si bien los restaurantes pueden optar por una gestión combinada de ambos recursos, dado que una de las funciones fundamentales del sumiller será la propia gestión de la carta-, esto no siempre ocurre. 
Es recuente el desconocimiento de las características de los vinos con que se cuenta en el restaurante y por tanto imposibilita al dependiente hacer una buena gestión de venta al no poder utilizar dichas características como argumento para la venta. (Ruiz, 2014)

Otro problema detectado es la reposición de los productos cuando se agotan, que en ocasiones demora bastante su reposición, lo que provoca pérdidas en las ventas si es un producto de alta demanda, además provoca la ausencia de vinos en la carta o que haya que reimprimirla a menudo. Esta situación se puede minimizar llevando un control adecuado de las ventas y los inventarios del almacén. Los principales proveedores de vinos Extracartas de este hotel son las firmas comerciales D Leones e ITH que poseen una variedad de vinos de diferentes países y marcas que posibilitan la confección de una buena carta que satisfaga los gustos de los clientes.

La bodeguilla del día es donde se encuentran los vinos necesarios para prestar un buen servicio al cliente sin dar innecesarios viajes a la bodega principal. Debe estar surtida de todos los vinos que se reflejan en la carta, exceptuando algunos vinos con una guarda complicada 0 relativamente delicada. La bodeguilla diaria se repone al terminar un servicio, ya sea de cena o de almuerzo, restituyendo las botellas consumidas ("Técnicas básicas del servicio de vinos", 2016).

\section{Conservación y almacenamiento}

Al analizar las condiciones reales de conservación y almacenamiento de los vinos de acuerdo se detectan problemas en el almacén central y en el Restaurante Especializado como son:

- El local del almacén central tiene una pared exterior con ventanas de cristales, que permite la entrada de la luz solar, lo cual perjudica la calidad del vino y acelera su envejecimiento.

- Posee un foco de luz bastante potente que incide directamente sobre los botelleros.

- No posee sistema de climatización, lo cual impide que mantenga una temperatura estable y 
adecuada para la conservación de los vinos; ya que la mayor parte del tiempo los vinos se encuentran expuestos a temperaturas excesivas.

- No se tiene control de la humedad

- La cava se encuentra situada a pocos metros de las neveras del almacén central, que son fuentes generadoras de vibraciones que afectan la estabilidad de los vinos, pues los estantes no poseen superficies que absorba las vibraciones.

- En el Restaurante Don Diego solo hay climatización en el horario de servicio, por lo que los vinos están expuestos a constantes cambios de temperatura.

- Los vinos blancos están colocados en refrigeradores, con unas temperaturas muy bajas y en forma vertical, lo cual afecta la correcta conservación de dichos vinos.

Es sistema más económico para guardar el vino, es la misma caja en la que llega desde la bodega de producción, la cual protege a los caldos de humedad, frío, calor y ruidos. Las grandes bodegas de restaurante utilizar estanterías, nichos o botelleros, fabricados a medida para los vinos y armarios específicos para los vinos blancos, rosados y espumosos. Una de las razones principales de tener una buena bodega es tener a mano el vino que guste en el momento que lo solicite el cliente. Otra razón es que a todo vino le hace bien reposar un mes o dos antes de beberlo. Los vinos maduros, en particular, no están en sus mejores condiciones si han estado moviéndose mucho y se han trasladado justo antes de beberlos ("Técnicas básicas del servicio de vinos", 2016).

El vino debe estar guardado en un lugar ventilado, protegido de la luz, vibraciones y a una temperatura constante. Pero veamos con detalle cinco aspectos fundamentales para conservar y guardar correctamente el vino ("¿Cómo se debe guardar y almacenar el vino?", 2020).

\section{- Humedad:}

Se recomienda que exista humedad en el ambiente, así como evitar los olores fuertes, e incluso los ruidos y los campos electromagnéticos (electrodomésticos). 


\section{- Movimientos}

El vino debe moverse lo menos posible, cuanto más añejo sea el vino más posibilidades de estropearse con los movimientos 0 vibraciones.

\section{- Luz}

Aunque a mucha gente le gusta exponer las botellas, hay que evitarlo, lo mejor es guardarlas en un lugar oscuro, la luz puede estropear un vino, especialmente los blancos.

\section{- Ventilación}

La humedad y la posición horizontal de almacenaje son responsables de la buena conservación del corcho, por este motivo debe haber una buena ventilación en la bodega, así evitaremos olores de moho 0 podredumbre.

\section{- Temperatura}

El vino debe guardarse a una temperatura constante de entre $5^{\circ} \mathrm{C}$ y $18^{\circ} \mathrm{C}$, siendo el punto óptimo entre $10^{\circ} \mathrm{C}-12^{\circ} \mathrm{C}$. Hay que evitar las oscilaciones bruscas y frecuentes de temperatura. Una bodega puede variar su temperatura, sin complicaciones para el vino, entre el invierno y el verano en $+/-5^{\circ} \mathrm{C}$, el problema viene si esta variación se produce semanalmente, o incluso a diario (noche-día). Los cambios de temperatura bruscos afectan principalmente al corcho, que cambia de tamaño y permite filtraciones de vino.

Debe colocarse la botella de manera lateral, la razón principal es para mantener el vino alejado del corcho. Cuando el vino está en contacto con el corcho, no se secará tan fácil. Esto no es necesariamente cierto para los vinos que planeas disfrutar en el corto plazo o para botellas que utilizan corchos artificiales, como de goma, plástico, vidrio o tapa rosca. Un corcho deteriorado puede arruinar con rapidez una botella de vino. Cuando un corcho natural se seca, puede agrietarse y permitir la exposición al aire, uno de los peores enemigos del vino. La exposición al aire a través de un corcho seco y agrietado puede dar al vino un aroma y un sabor desabridos ("Administración de bares y restaurantes: Consejos para el correcto almacenamiento del vino", 2017).

La mayoría de vinos rojos debe mantenerse a no menos de trece grados para largos periodos de almacenamiento, pero hay algunas excepciones. Por ejemplo, el vino 
rojo con mucho cuerpo puede mantenerse a altas temperaturas y los vinos rojos más ligeros pueden mantenerse a alrededor de $10^{\circ} \mathrm{C}$. En general, mantén los vinos rojos debajo de los $21^{\circ} \mathrm{C}$ para obtener mejores resultados. El vino blanco se sirve mejor a temperaturas ligeramente inferiores que el rojo. Se aplica la misma regla básica. Mientras más cuerpo tenga el vino, puedes almacenarlo a mayor temperatura dentro del rango sugerido, que es alrededor de los 4 y 13ํ C ("Administración de bares y restaurantes: Consejos para el correcto almacenamiento del vino", 2017).

Caracterización y diseño de la carta de vinos

El restaurante Don Diego cuenta con una carta de vinos con una presentación elegante que incluye una gran variedad de productos en su oferta con representación de los diferentes estilos de vinos y de los diferentes países productores de más reconocimiento.

Al analizar la carta de vinos del Restaurante Don Diego del Hotel Sol Cayo Coco, se detecta la necesidad de realizar algunas mejoras en su diseño y contenido que propicien una mejor gestión de venta de los vinos, de acuerdo con los aspectos técnicos y de diseño que deben cumplir las cartas de vino es necesario realizar cambios a los siguientes elementos:

- La letra fuente debe ser legible; por lo que debe emplearse una fuente menos artística, y de mayor tamaño para lograr una fácil comprensión del texto. (Es recomendable usar el estilo Arial.

- Los vinos deben presentar en la información el mismo orden para; el nombre del vino, la uva y la denominación de origen para una mejor apreciación por parte del cliente.

- Debe presentarse la añada de los vinos como un dato importante para la elección del vino por parte del cliente. En el caso de esta carta en ningún vino está reflejada la añada.

- En algunos casos se omite la denominación de origen y la variedad de la uva del vino que es otro dato que debe aparecer como información al cliente.

- La carta tiene vinos con D.O mezclados con otros que no lo poseen. 
- Los vinos blancos se encuentran en mayor número, cuando según el criterio de especialistas en una carta deben predominar los vinos tintos.

- La carta está muy extensa de acuerdo al menú de comida que ofrece el restaurante.

- Dentro de la carta se pueden encontrar vinos blancos jóvenes, tintos y rosados con añadas que ya por su ciclo de vida no están en condiciones de ser consumidos.

- La variedad de ofertas de vinos franceses es muy pobre, (solo 2 exponentes de 52 variedades que posee la carta) siendo estos de muy buena calidad y teniendo gran aceptación.

- Los vinos espumosos deben tener mayor representación, por ser de acuerdo con el diagnóstico realizado, el producto de más popularidad en la carta.

- Hay vinos en la carta que están agotados en la cava, lo que perjudica la imagen del restaurante.

Entre los factores a tener en cuenta para la confección de la carta de vinos se encuentras: la ubicación del restaurante, está ubicado en zonas productoras de vino, la selección de vinos de la propia zona debe ser amplia para satisfacer a clientes foráneos y locales; la carta de vinos ha de contener variedad de vinos, de distintas tipologías y orígenes; la selección de vinos se ha de realizar teniendo en cuenta la oferta gastronómica; la carta de vinos ha de reflejar la ambición y aspiraciones del restaurante, la inclusión de una selección de vinos selectos y de prestigio dan categoría al establecimiento y sirve para vender aquello que el profesional considere oportuno; tiene que ser fácilmente reemplazable para que la introducción de cambios de añada y nuevos vinos no sea complicada; los vinos se deben rotar, y su rotación se facilita, entre otras técnicas, mediante ofertas y la venta de vinos por copas. Los vinos se deben presentar: DO. o zona de producción; nombre del vino y de la bodega; tipo de vino; añada; variedades y precio por copa y botella (García, 2019).

Para la elección de las referencias de la carta de vinos se deben considerar: los precios de compra y 
venta, una de las cosas más criticadas son los precios en las cartas. Comprar barato no significa comprar bien; la diversidad geográfica es importante, existen clientes que no demandan un vino en concreto, sino una región en concreto; las variedades de uva son otro elemento clave en la carta de vinos. Una buena opción es contar con referencias que tengan diferencias varietales para allegar al mayor número posible de clientes y las marcas más mediáticas que son más fáciles de vender, pero las menos conocidas ofrecen al cliente una cierta singularidad. No es recomendable repetir muchas referencias de una misma marca ("Veinte items para crear una carta de vinos" 2015).

\section{Servicio de los vinos}

En el restaurante Don Diego se observan detalles negativos que deterioran los estándares de calidad esperados como son:

- No existen suficientes cartas de vino (solo existen dos) para un salón en el que entran aproximadamente 12 mesas al mismo tiempo.
- El personal de servicio y de almacenes no está capacitado en lo referente al conocimiento del producto y a las técnicas de mercado.

- La carta de vinos no está expuesta en la entrada del restaurante.

El formato y la disposición de la carta de vinos. Es una de las partes más importantes, pues debe combinar comodidad y atractivo a la vez. La carta debe ser dinámica o con posibilidades de cambios para que no resulte aburrida ("Veinte items para crear una carta de vinos" 2015).

Se debe preparar el personal seleccionado para llevar a cabo el proceso en aspectos afines, tales como: calidad, métodos y herramientas para recopilar información, técnicas de trabajo en grupo y desarrollo de estrategias de mejora, gestión de ventas, servicio de los vinos, maridaje, entre otros. Este es uno de los principales pasos donde se deberá garantizar el compromiso del personal, se comienza por la alta dirección; si no se logra este compromiso no se podrá asegurar la calidad del proceso de medición ni la aplicación 
integral del procedimiento, sólo con

esta premisa cumplida se facilitará la ruptura de trabas paradigmáticas (Rodríguez, 2019).

\section{Medidas para el mejoramiento} de las ventas de vinos en

\section{extracartas}

1. Llevar control en el almacén de la rotación de los vinos según su ciclo de vida de acuerdo a las características de los productos, lo que evitaría pérdidas por deterioro de los vinos.

2. Estudiar los volúmenes de consumo de los diferentes vinos para lograr el abastecimiento ininterrumpido de los productos ofertados en la carta.

3. Superación del personal de almacenes que trabaja con las bebidas; para lograr los conocimientos requeridos sobre el tema.

4. Eliminar la entrada de la luz solar al almacén de vinos.

5. Cambiar el foco de luz del almacén por otro menos potente; pues los vinos envejecen prematuramente por el exceso de iluminación.
6. Climatizar el almacén de vinos.

7. Colocar los estantes de vinos sobre superficies de goma que absorba las vibraciones producidas por la cercanía de las neveras de refrigeración.

8. Colocar las botellas de vino blanco del Restaurante Don Diego en estanterías de manera horizontal para garantizar su hermeticidad y la correcta humidificación del corcho.

9. Colocar la carta de vinos en algún sitio a la entrada del restaurante y en la zona del Lobby para mejorar su publicidad.

10. Aumentar la cantidad de cartas para el servicio del restaurante.

11. Superación del personal del restaurante en el tema de sommeliería.

12. Darles solución a los vinos eliminados de la carta pasándolos a todo incluido, a cocina, o ponerlos en ofertas especiales según corresponda de acuerdo con el estado y características de cada uno. 


\section{Conclusiones}

- En el hotel existen problemas de gestión en el almacén por la existencia de vinos que ya no están aptos para la venta lo que representa pérdida de recursos.

- El personal que atiende esta área de servicio no posee la capacitación necesaria para el manejo de vinos.

- El almacén no cuenta con las características de conservación idóneas para la preservación de los vinos existentes.

- La carta de vinos del servicio extracarta del Restaurante Especializado Don Diego no está acorde con el menú, cuenta con poca información y no se realiza una constante actualización según la disposición de la cava de vinos.

\section{Bibliografía}

Administración de bares y restaurantes: Consejos para el correcto almacenamiento del vino. (2017). Recuperado de:

https://www.gediscovery.edu. pe/blog/c/noticias/
¿Cómo se debe guardar y almacenar el vino?. (2020). Recuperado de: https://www.vinetur.com/

Gil, I., Berenguer, G., Ruiz, M.E. (2009). Instrumentos de promoción de los vinos en los restaurantes de alto nivel. Investigaciones Europeas de Dirección y Economía de la Empresa,15 (2), 63-76, ISSN: 1135-2523.

Instituto Nacional Tecnológico, Dirección General de formación profesional. (2016). Manual del protagonista. Técnicas básicas de servicio de vinos. Nicaragua.

Nieto, J. L., Román, I.M., Bonillo, D., Paulova, N. (2016). El turismo a nivel mundial. International Journal of Scientific Management and Tourism, 2 (1), 129-144.

García, N. (2019). La Carta de Vinos: Estructura y factores a tener en cuenta. Recuperado el 21 de octubre de 2020, de https://www.gourness.com/bl og/aprende/servicio del vino y su conservación/

Rodríguez, V. (2019). Procedimiento para la gestión de venta de vinos en hoteles que emplean la modalidad todo incluido. Trabajo de grado, Ingeniería Industrial, Universidad de Holguín, Holguín, Cuba. 
Ruiz, A. (2014). Estrategia de venta para vinos extracarta en el restaurante especializado "Garibaldi" del hotel Playa Cayo Santa María. Trabajo de grado, Licenciatura en Turismo, Universidad Central Marta Abreu de las Villas, Santa Clara, Cuba.

Veinte items para crear una carta de vinos. (2015). Recuperado el 21 de octubre de 2020, de https://sumillercampo.wordpr ess.com/

Venegas, H. (2005). Un triángulo cubano: turismo, patrimonio, comunidad. Temas, 43, 5666. 